

Scand J Work Environ Health 2004;30(4):331-333

https://doi.org/10.5271/sjweh.803

Issue date: Aug 2004

Prevention of work-related decompression illness events by detection of a cardiac right-to-left shunt

by Kütting B, Tomandl B, Drexler H

Affiliation: Institute and Outpatient Clinic of Occupational, Social and Environmental Medicine, University of Erlangen-Nurember, Schillerstrasse $25+29$, D-91054 Erlangen, Germany. birgitta.kuetting@ipasum.uni-erlangen.de

Key terms: cardiac right-to-left shunt; cardiac shunt; case report; decompression illness; occupational medicine; patent foramen ovale; prevention; professionals under hyperbaric conditions; work

This article in PubMed: www.ncbi.nlm.nih.gov/pubmed/15458018 


\title{
Prevention of work-related decompression illness events by detection of a cardiac right-to-left shunt
}

\author{
by Birgitta Kütting, MD, ${ }^{1}$ Bernd Tomandl, MD, ${ }^{2}$ Hans Drexler, $M D^{1}$
}

\begin{abstract}
Kütting B, Tomandl B, Drexler H. Prevention of work-related decompression illness events by detection of a cardiac right-to-left shunt. Scand J Work Environ Health 2004;30(4):331-333.

A 44-year-old tunnel worker was studied who suffered from several unexplained decompression illness events for almost 15 years. This caisson worker was affected after standard pressure profiles that did not cause symptoms of decompression illness in his colleagues on the same shift. Transesophageal echocardiography revealed an atrial septal defect (grade II) in this otherwise healthy man. Cranial magnetic resonance imaging showed ischemic brain lesions. Among divers, patent foramen ovale, the most common cause of cardiac right-toleft shunts, was shown to increase the risk for decompression illness events by a factor of 4.5 and to double the risk of ischemic brain lesions. Hyperbaric workers with symptoms of unexplained decompression illness, even if they are only slight, should immediately be transferred to a cardiologist so that a cardiac right-to-left shunt will not be overlooked.
\end{abstract}

Key terms cardiac right-to-left shunt, case report, decompression illness, patent foramen ovale, professionals under hyperbaric conditions, occupational medicine.

Patent foramen ovale is a remnant of fetal circulation and is the most common cause of a right-to-left shunt (1). Oxygenated placental blood enters the right atrium via the inferior vena cava and crosses the valve of the foramen ovale to enter the systemic arterial system. At birth, pulmonary vascular resistance and right-sided cardiac pressure drop with a reversal of the right-atriumto-left-atrium pressure gradient. The flap of the foramen ovale closes against the artrial septum, fusion generally occurring within the first 2 years of life (2). Less frequent causes of right-to-left shunts include artrial septal defects and pulmonary arteriovenous malformations.

It has been shown that patent foramen ovale is a risk factor for the development of multiple brain lesions among sports divers, as shown in magnetic resonance imaging (MRI). The brain lesions are presumably caused by paradoxical bubble emboli $(3,4)$. Schwerzmann et al (4) observed twice as many ischemic brain lesions in divers with patent foramen ovale than in those without the abnormality. Spinal or cerebral symptoms of decompression sickness were also shown to occur in 4 of 13 divers with and 4 of 39 divers without patent foramen ovale. Arterial gas embolism occurred in 4 of 13 divers with and 2 of 39 divers without patent foramen ovale. In a logistic regression model, patent foramen ovale increased the risk of decompression illness events in divers by 4.5 during all dives.

In the general population, patent foramen ovale is a common finding; the fusion is incomplete in approximately one-fourth of the general population, resulting in an oblique slit-like defect (5). Two autopsy studies determined an incidence between $27.3 \%$ (1) and 35\% (6) for patent foramen ovale. Small patent foramen ovales probably close spontaneously throughout adult life, as there is a reduction in prevalence from $34 \%$ in the first three decades of life to $20 \%$ in the ninth and tenth decades (1).

Thus far, cardiac right-to-left shunts, especially patent foramen ovale, have been implicated in several pathological processes, including migraine with aura $(7,8)$, paradoxical embolism in cryptogenic stroke, and venous-to-arterial gas embolism in serious or less serious forms of decompression illness $(3,4,9-13)$.

Because most people with patent foramen ovale show no obvious clinical signs, diagnosis is usually only attempted following an unexplained incidence

1 Institute and Outpatient Clinic for Occupational, Social and Environmental Medicine, University of Erlangen-Nuremberg, Erlangen, Germany.

2 Department of Neuroradiology, University of Erlangen-Nuremberg, Erlangen, Germany.

Correspondence to: Dr Birgitta Kütting, Institute and Outpatient Clinic of Occupational, Social and Environmental Medicine, University of Erlangen-Nuremberg, Schillerstrasse $25+29$, D-91054 Erlangen, Germany. [E-mail: birgitta.kuetting @ipasum.uni-erlangen.de] 
of decompression illness. Transesophageal echocardiography is currently considered the reference standard for the diagnosis of patent foramen ovale since it allows direct imaging of the interatrial septum and saline contrast shunting through a patent foramen ovale. If color flow Doppler or peripheral saline contrast injection detect right-to-left flow during normal respiration, it is termed a resting patent foramen ovale (2). Data of methodological studies suggest that cough or the Vasalva maneuver increase the sensitivity of detecting transesophageal patent foramen ovale and that the contrast injections via the femoral vein approach are superior to the antecubital route $(14,15)$.

Transthoracic echocardiography with contrast injection can be used to diagnose patent foramen ovale as well, but it has a sensitivity of up $80 \%$ when compared with that of transesophageal echocardiography. Thus negative transthoracic echocardiography does not exclude the existence of patent foramen ovale, but a diagnostic transthoracic echocardiography may avoid the need for a transesophageal echocardiography (16).

In contrast to patent foramen ovales, atrial septal defects are more likely to have already been diagnosed since the signs and symptoms are usually more obvious. The symptoms include heart murmur, cyanosis, and faintness.

In this paper, we report the findings from a tunnel worker suffering from several unexplained decompression illness events over a period of almost 15 years. Transesophageal echocardiography revealed an atrial septal defect (grade II).

To the best of our knowledge, this case study is the first in occupational medicine to report on the relation between cardiac right-to-left shunt and decompression illness.

\section{Case report}

In 1988, a 44-year-old caisson worker first complained several times of bends after working under conditions of compressed air (approximately 2 bars). Two years later he felt faint after the decompression phase and suffered from circulation problems for about 6 weeks. In nearly all the events the man was transferred to a hyperbaric unit for recompression treatment. In October 2002, while working on tunnel construction in Switzerland (42 minutes under compressed air of 2.7 bars), he suffered from paraesthesia, burning, and pain in the right leg $10 \mathrm{~min}-$ utes after the end of the decompression phase. In the next hour, all of these sensitizations worsened and were followed by ataxia. Transfer to a hyperbaric facility was immediately organized; on the way all the symptoms had disappeared spontaneously after 4 hours. But, as a preventive measure, recompression therapy was applied for 6 hours. The caisson worker also reported past blurred vision after work under conditions of compressed air. The pressure profiles were standard, and none of his colleagues was affected by decompression illness events. The man's clinical examinations, including pulmonary function tests, an electrocardiogram, chest X-rays, and blood pressure, had always been normal. His history was negative for internal, especially cardiological, disease.

He reported that he had been a nonsmoker for 8 years. Before he gave up smoking, he consumed 25 cigarettes a day over a period of 17 years. He also denied any intake of drugs.

After the last event of decompression illness, a complete medical examination was performed that included transesophageal echocardiography and MRI. The transesophageal echocardiography revealed an atrial septal defect (grade II). Direct shunting of contrast bubbles into the left atrium through the artrial septal defect was detected during the application of the Valsalva maneuver.

MRI revealed a total of 18 not well delineated hyperintense lesions (13 on the left side, and 5 on the right side) predominantly in the periventricular white matter.

\section{Discussion}

In 1986, Wilmshurst et al (17) were the first to report on paradoxical gas embolism in a scuba diver with an artrial septal defect. In 1989, after observation of 30 patients with a history of decompression illness, Moon et al (10) assumed that patent foramen ovale may be a risk factor for the development of decompression illness in divers. Since then, numerous publications have confirmed this observation and reported an increased risk of the development of decompression illness, as well as multiple brain lesions, as shown by MRI if a cardiac right-to-left shunt was present $(3-5,7,8,10-13,18-23)$. Although the role of patent foramen ovale in the pathogenesis of decompression illness has been established, the magnitude of the associated risk still remains controversial (24). In contrast, the pathological significance of brain lesions is somewhat unclear (3).

Although patent foramen ovale is common, adverse consequences arise infrequently, but have been implicated in several pathological processes such as venous-toarterial gas embolism in serious forms of decompression illness. For diving, it was hypothesized that venous gas bubbles liberated after the diver's rise to the surface may enter the systemic circulation through a patent foramen ovale and embolize into the central system ("paradoxical embolism") (11). Right-to-left interatrial shunting can occur when right arterial pressure is increased. According to Schwerzmann \& Seiler (4), patent foramen ovale is probably the most prevalent pathway out of all the possibilities for the arterialization of venous gas bubbles. If no cardiac or pulmonary shunt is present, small volumes of venous gas bubbles are tolerated well and entrapped in and exhaled from the lungs (25). 
Decompression illness can occur during flights, from altitude chamber exposure, from professional deep-sea diving, and from working in pressurized tunnels or caissons for bridge-building projects.

The term decompression illness is used for describing all decompression disorders with bubbles as the initiator. It is used as a collective term for decompression sickness, resulting from the growth of gas nuclei in predominantly fatty tissue, and also for arterial gas embolism, due to gas invasion into systemic circulation (4). A clear clinical distinction between arterial gas embolism and decompression sickness is not always possible; therefore, the term decompression illness is preferred.

Clinical manifestations of decompression illness are traditionally classified as minor (type I) or major (type II) symptoms. Minor symptoms include muscoloskeletal pain and "skin bends" with rash, pruritus, and painless swelling of the skin due to lymphatic obstruction. Major decompression illness includes neurological (spinal and cerebral decompression illness) or cardiorespiratory disorders. The signs of spinal decompression illness are limb weakness, cutaneous sensory level and impaired bowel or bladder control and the signs of cerebral decompression illness are blurred vision, dysarthria, hemiplegia, or loss of consciousness (3).

The high prevalence of a cardiac right-to-left shunt, especially of a foramen ovale in the general population, stresses the relevance of this relation for the field of occupational medicine, especially for occupational medical care for the group of hyperbaric workers. Therefore, company physicians taking care of hyperbaric professionals should be aware of the potential risk of decompression illness for workers with a cardiac right-to-left shunt. Hyperbaric workers with symptoms of unexplained decompression illness, even if they are only slight, should immediately be transferred to a cardiologist so that a cardiac right-to-left shunt will not be overlooked. If the diagnosis of a cardiac right-to-left shunt is confirmed, working under pressurized conditions should be stopped immediately.

\section{References}

1. Hagen PT, Swcholz DG, Edwards WD. Incidence and size of patent foramen ovale during the first decades of life: an autopsy study of 965 normal hearts. Mayo Clin Proc 1984;59:17-20.

2. Kerut EK, Norfleet WT, Plotnick GD, Giles TD. Patent foramen ovale: a review of associated conditions and the impact of physiological size. J Am Coll Cardiol 2001;38:613-23.

3. Schwerzmann M, Seiler C, Lipp E, Guzman R, Lövblad KO, Kraus M, et al. Relation between directly detected patent foramen ovale and ischemic brain lesions in sport divers. Ann Intern Med 2001;134:21-4.

4. Schwerzmann M, Seiler C. Recreational scuba diving, patent foramen ovale and their associated risks. Swiss Med Wkly 2001;131:365-74.

5. Langton P. Patent foramen ovale in underwater medicine. South Pac Underwater Med J 1996;26(3):186-91.

6. Thompson T, Evans W. Paradoxical embolism. QJM 1930;23:135-52.

7. Wilmshurst PT, Nightgale S, Walsh KP, Morrison WL. Effect on migraine of closure of cardiac right-to-left shunts to prevent recurrence of decompression illness or stroke or for haemodynamic reasons. Lancet 2000;365:1648-51.

8. Wilmshurst $\mathrm{P}$, Nightingale $\mathrm{S}$. Relationship between migraine and cardiac and pulmonary right-to-left shunts. Clin Sci 2001;100:215-20.

9. Foster PP, Boriek AM, Butler BD, Gernhardt ML, Bove AA. Patent foramen ovale and paradoxical systemic embolism: a bibliographic review. Aviat Space Environ Med 2003;74(6 Pt 2):B1-64.

10. Moon RE, Camporesi EM, Kisslo JA. Patent foramen ovale and decompression sickness in divers. Lancet 1989;1:513-4.

11. Wilmshurst PT, Byrne JC, Webb-Peploe MM. Relation between interatrial shunts and decompression sickness in divers. Lancet 1989;2:1302-6.

12. Wilmshurst P, Bryson P. Relationship between clinical features of neurological decompression illness and its causes. Clin Sci 2000;99:65-75.

13. Wilmshurst PT, Pearsom MJ, Walsh KP, Morrison WL, Bryson P. Relationship between right-to-left shunts and cutaneous decompression illness. Clin Sci 2001;100:539-42.

14. Gin KG, Huckell VF, Pollick C: Femoral vein delivery of contrast medium enhances transthoracic echocardiographic detection of patent foramen ovale. J Am Coll Cardiol 1993;22:1994-2000.

15. Hamann GF, Schatzer-Klotz D, Frohling G, Strittmatter M, Jost V, Berg G, et al. Femoral injection of echo contrast medium may increase the sensitivity of testing for a patent foramen ovale. Neurology 1998;50:1423-8.

16. Alp N, Clarke N, Banning AP. How should patients with patent foramen ovale be managed. Heart 2001;85:242-4.

17. Wilmshurst PT, Ellis BG, Jenkins BS. Paradoxical gas embolism in a scuba diver with artrial septal defect. BMJ 1986;293(6557):1277.

18. Wilmshurst PT, Treacher DF, Crowther A, Smith SE. Effects of a patent foramen ovale on arterial saturation during exercise and on cardiovascular responses to deep breathing, Valsalva manoeuvre, and passive tilt: a relation to history of decompression illness in divers. Br Heart J 1994;71:229-31.

19. Wilmshurst P. Patent foramen ovale and decompression illness. South Pac Underwater Med J 1996;26(3):82-3.

20. Turner M. Patent foramen ovale and decompression illness in divers. Lancet 1996;Nov 30, 348 (9040):1515.

21. Johnston PR, Broome JR, Hunt PD, Benton PJ. Patent foramen ovale and decompression illness in divers. Lancet 1996; Nov 30, 348 (9040):1515.

22. Wilmshurst P, Walsh K, Morrisson L. Patent foramen ovale and decompression illness in divers. Lancet 1997; Jan 25, 349(9047):288.

23. Laden GD. Patent foramen ovale and decompression illness in divers. Lancet 1997;Jan 25, 349(9047): 288.

24. Francis J, Mitchell S. Pathophysiology of decompression sickness. In: Brubakk A, Neuman T. The physiology and medicine of diving. 5th ed. Philadelphia (PA): WB Saunders Ltd; 2003. p 533-4.

25. Butler BD, Hills BA. The lung as a filter for microbubbles. J Appl Physiol 1979;47:537-43.

Received for publication: 6 November 2003 